\title{
Microwave Irradiated Cross Coupling of Carboxylic Acids and Crotyl Bromides: Efficient Application to Make Arachidonic Acid Esters
}

\author{
Mohammad Al-Masum*, Arpona Hira \\ Department of Chemistry, Tennessee State University, Nashville, USA \\ Email: *malmasum@tnstate.edu
}

How to cite this paper: Al-Masum, M. and Hira, A. (2018) Microwave Irradiated Cross Coupling of Carboxylic Acids and Crotyl Bromides: Efficient Application to Make Arachidonic Acid Esters. International Journal of Organic Chemistry, 8, 341-348. https://doi.org/10.4236/ijoc.2018.84026

Received: September 20, 2018

Accepted: November 19, 2018

Published: November 22, 2018

Copyright $\odot 2018$ by authors and Scientific Research Publishing Inc. This work is licensed under the Creative Commons Attribution International License (CC BY 4.0).

http://creativecommons.org/licenses/by/4.0/

Open Access

\begin{abstract}
A microwave irradiated palladium-catalyzed reaction of carboxylic acids and crotyl type bromides creates series of esters in good to high yields. This facile ester synthesis then is applied to make esters from arachidonic acid, salicylic acid, folic acid, and aspirin efficiently.
\end{abstract}

\section{Keywords}

Arachidonic Acid Esters by Cross Coupling, Microwave

\section{Introduction}

Esters are common chemicals with extensive applications in medicine, biology, chemistry, and material sciences [1]-[7]. Esters used not only as solvents but also are in perfumes, essential oils, agriculture and food flavorings, antioxidant, plastics, detergents, and for many other purposes. Isoamyl acetate (odor of banana), ethyl butanoate (odor of mango), methyl 2-methylbutanoate (odor of pineapple), vitamin C, cocaine, etc., are some common interesting examples occurring in the nature. The enormous use of transition metal complexes to activate organic molecules makes them viable visions for developing catalytic processes with high selectivity and atom economy. This work focusses to find a facile way of making esters by microwave irradiated cross-coupling reaction of carboxylic acids, allyl type halides, in the presence of palladium-catalyst (Scheme 1) and apply that effective cross coupling method to synthesize arachidonic acid esters, and folic acid esters, etc.

\section{Results and Discussion}

Recently, $\mathrm{PdCl}_{2}\left(\mathrm{~d}^{\mathrm{t}} \mathrm{bpf}\right)$ complex has been successfully employed as a catalyst in 


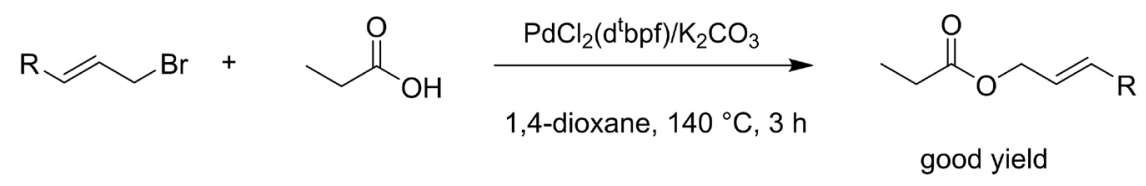

Scheme 1. Propionic acid ester from Propionic acid and alkyl halides.

various organic transformations involving potassium organotrifluoroborates [8] [9] [10] [11] [12]. The higher cone angle of P-Pd-P in $\mathrm{PdCl}_{2}\left(\mathrm{~d}^{\mathrm{t} b p f}\right)$ may be improve its effectiveness as a catalyst. In order to see further application of this palladium complex, we thought to explore cross coupling reactions of carboxylic acids with 3-Bromo-1-phenyl-1-propene $\mathbf{2 a}$ and 1-bromo-2-butene $\mathbf{2} \mathbf{b}$. We began our study with 3-bromo-1-phenyl-propene $2 \mathrm{a}$ and various carboxylic acids as substrates with the load of 3 mole $\% \mathrm{PdCl}_{2}\left(\mathrm{~d}^{\mathrm{t}} \mathrm{bpf}\right)$. The reactions worked well when microwaved for $30 \mathrm{~min}$. We turned our attention to optimization the reaction conditions and found 0.5 mole $\%$ catalyst works quite good with $3 \mathrm{~h}$ reaction time.

The results with 3-bromo-1-phenyl-1-propene 2a summarize in Figure 1. In case of cinnamic acid best result obtained with 3 mole $\% \mathrm{PdCl}_{2}\left(\mathrm{~d}^{\mathrm{t}} \mathrm{bpf}\right)$ (Entry 10, Figure 1).

We also unzipped the cross coupling of carboxylic acids with 3-bromo-2-butene $2 \mathrm{~b}$. The reactions went very sluggish but with 3.0 mole \% catalyst the desired products form in moderate yields. The results with 3 -bromo-2-butene $2 \mathrm{~b}$ summarize in Figure 2. In case of hexanoic acid and 2-octenoic acid, 0.5 mole $\%$ of catalyst worked well when treated with 1-bromo-2-butene $\mathbf{2 b}$ (Figure 2, Entries $2,3)$.

It is a further object of the investigation to see the application of these methods and make the esters from arachidonic acid, folic acid, salicylic acids, etc. In fact, arachidonic acid, folic acid, salicylic acid, all form the corresponding esters when treated with halides $2 \mathrm{a}$ and $\mathbf{2 b}$. Examples such as $4 \mathrm{a}, \mathbf{4 b}, \mathbf{4 c}$, and $4 \mathrm{~d}$ present in Scheme 2.

Our goal is to examine the significant biological effect of these new esters and report in due courses. Arachidonic and folic acid esters are useful biologically active compounds [13] [14] [15] [16] [17]. The probable catalytic cycle for this new transformation proposes in Scheme 3. This new method is eco-friendly, atom-economy, and sustainable green chemistry synthetic process. This new process will have potential value for complex ester synthesis.

\section{Procedure}

The synthesis of ester $3 \mathbf{a}$ from propionic acid 1a and 3-bromo-1-phenyl-1propene $2 \mathrm{a}$ is a representative one. A dry clean microwave vial was loaded with potassium carbonate $(0.207 \mathrm{~g}, 1.5 \mathrm{mmol}), \mathrm{PdCl}_{2}\left(\mathrm{~d}^{\mathrm{t} b p f}\right)(0.002 \mathrm{~g}, 0.0025 \mathrm{mmol})$, then capped the vial with septum and flushed with argon. After adding propionic acid (37.5 $\mu \mathrm{L}, 0.5 \mathrm{mmol})$, 3-bromo-1-phenyl-propene $2 \mathrm{a}(82.0 \mu \mathrm{L}, 0.55 \mathrm{mmol})$ via micro syringe, and 1,4-dioxane $(5.0 \mathrm{~mL})$ in the microwave reaction vial, the result- 
ing mixture was irradiated at $140^{\circ} \mathrm{C}$ for $3 \mathrm{~h}$. The crude reaction product filtered through sintered funnel and concentrate. For purification, the crude product passed through alumina using hexane/dichloromethane (100/1) as eluents. The purified product $3 \mathrm{a}$ obtained was $87 \%$ in yield (Figure 1, entry 1). Compound 3a, ${ }^{1} \mathrm{H}$ NMR $\left(\mathrm{CDCl}_{3}, 400 \mathrm{MHz}\right) \delta 7.24(\mathrm{~m}, 5 \mathrm{H}), 6.57(\mathrm{~d}, \mathrm{~J}=15.8 \mathrm{~Hz}, 1 \mathrm{H}), 6.21$ $(\mathrm{dt}, \mathrm{J}=6.44 \mathrm{~Hz}, 1 \mathrm{H}), 4.66(\mathrm{~d}, \mathrm{~J}=6.48 \mathrm{~Hz}, 2 \mathrm{H}), 2.30(\mathrm{q}, \mathrm{J}=7.56 \mathrm{~Hz}, 2 \mathrm{H}), 1.09(\mathrm{t}, \mathrm{J}$ $=7.56 \mathrm{~Hz}, 3 \mathrm{H}) ;{ }^{13} \mathrm{C} \mathrm{NMR}\left(\mathrm{CDCl}_{3}, 100 \mathrm{MHz}\right)$

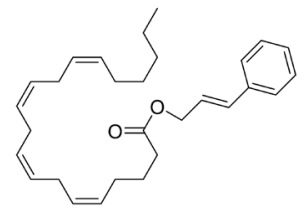

4a, Arachidonic acid ester

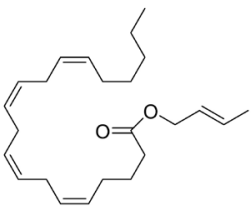

4b, Arachidonic acid ester

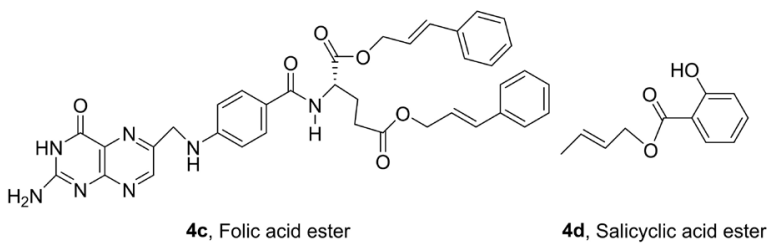

Scheme 2. New esters from arachidonic, folic, and salicylic acids.

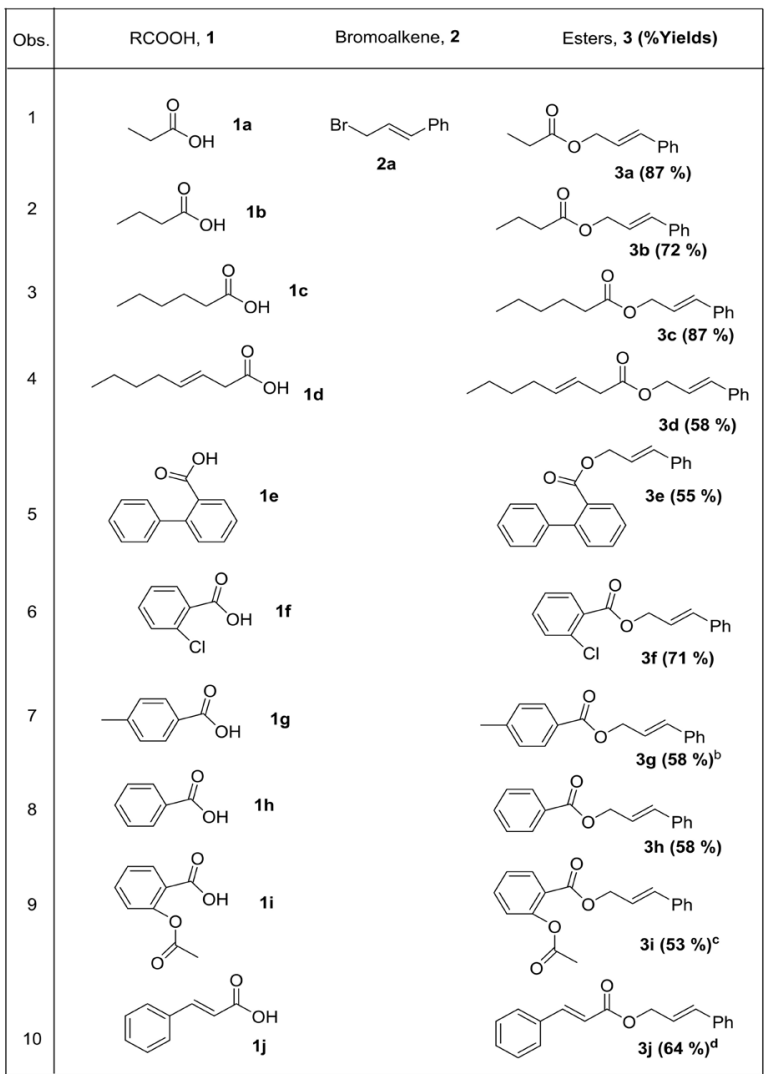

Figure 1. Esters from cross coupling of carboxylic acids and bromoalkenes ${ }^{\mathrm{a}}$. ${ }^{\mathrm{a}}$ All yields are isolated yields; ${ }^{\mathrm{b}} \alpha$-adduct is minor product; ${ }^{\mathrm{c}}$ Mixture of E; and Z ${ }^{\mathrm{d}}$ Compound $3 \mathrm{j}$ formed by adding 3 mole $\% \mathrm{PdCl}_{2}\left(\mathrm{~d}^{\mathrm{t}} \mathrm{bpf}\right)$. 


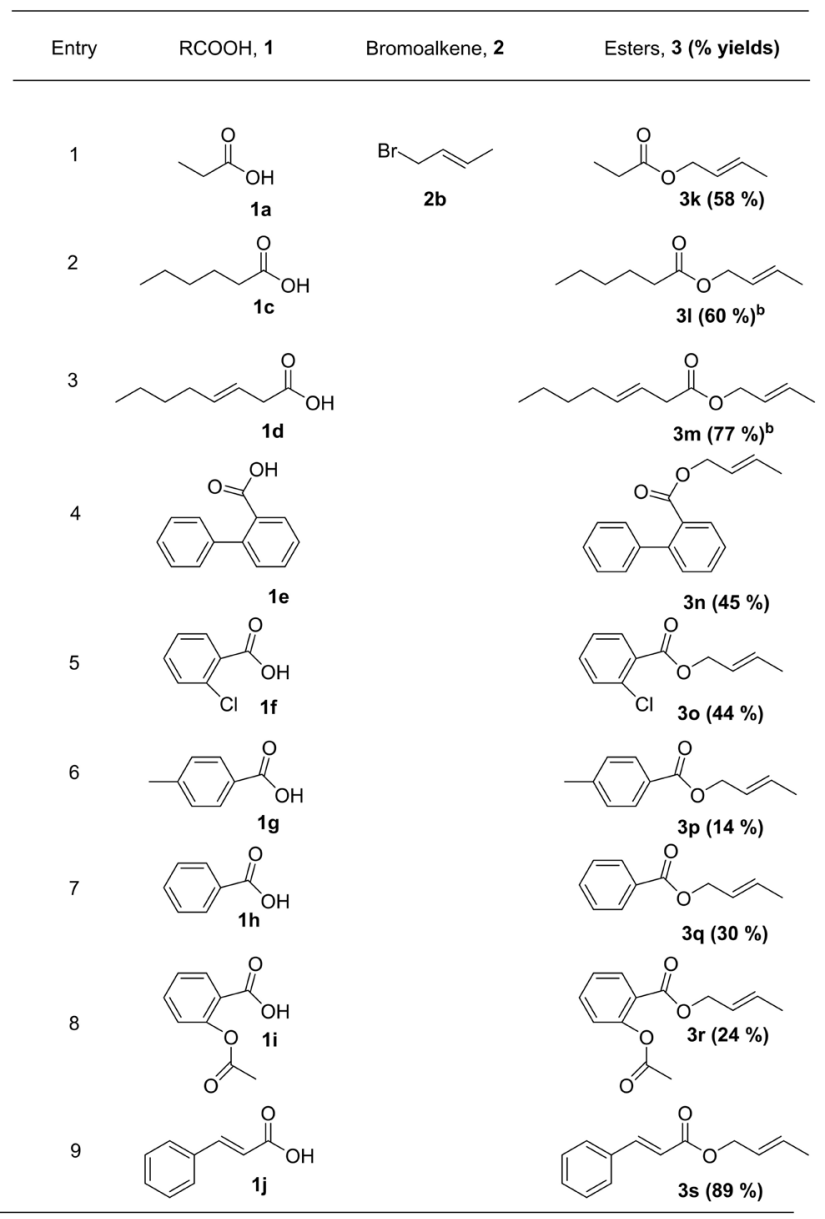

Figure 2. Esters from cross coupling of carboxylic acids and 3-bromo2-butene $2 \mathrm{~b}^{\mathrm{a}}$. ${ }^{\mathrm{a}}$ Reactions run with the load of 3 mole $\% \mathrm{PdCl}_{2}\left(\mathrm{~d}^{\mathrm{t} b p f}\right) ;{ }^{\mathrm{b}} 3 \mathrm{l}$ and 3 $\mathrm{m}$ products form with 0.5 mole \% load of $\mathrm{PdCl}_{2}\left(\mathrm{~d}^{\mathrm{t} b p f}\right)$.

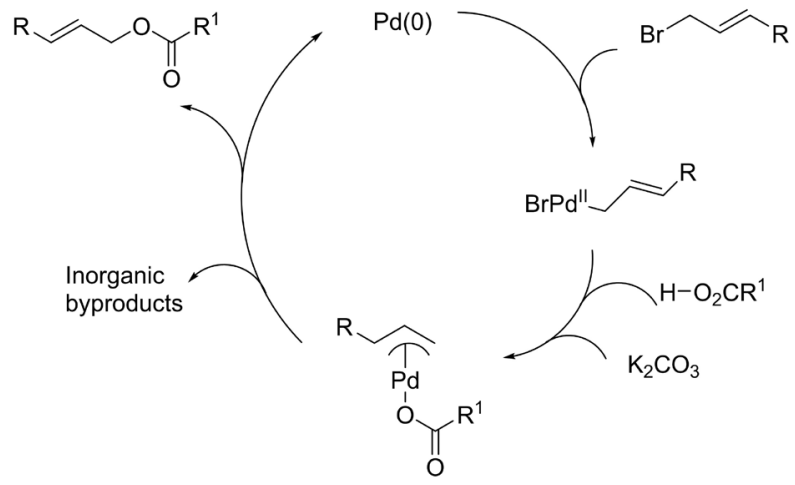

Scheme 3. Catalytic cycle of esters from $\mathrm{R}^{1} \mathrm{COOH}$ and crotyl halide.

\subsection{Figure 1}

Compound 3a, ${ }^{1} \mathrm{H}$ NMR $\left(\mathrm{CDCl}_{3}, 400 \mathrm{MHz}\right) \delta 7.24(\mathrm{~m}, 5 \mathrm{H}), 6.57(\mathrm{~d}, \mathrm{~J}=15.8 \mathrm{~Hz}$, $1 \mathrm{H}), 6.21(\mathrm{dt}, \mathrm{J}=6.44 \mathrm{~Hz}, 1 \mathrm{H}), 4.66(\mathrm{~d}, \mathrm{~J}=6.48 \mathrm{~Hz}, 2 \mathrm{H}), 2.30(\mathrm{q}, \mathrm{J}=7.56 \mathrm{~Hz}$, $2 \mathrm{H}), 1.09(\mathrm{t}, \mathrm{J}=7.56 \mathrm{~Hz}, 3 \mathrm{H}) .{ }^{13} \mathrm{C} \mathrm{NMR}\left(\mathrm{CDCl}_{3}, 100 \mathrm{MHz}\right) \delta 174.2,136.2,134.1$, $128.6,128.5,128.0,126.6,123.3,64.9,27.6,9.1$; 
Compound 3b, ${ }^{1} \mathrm{H}$ NMR $\left(\mathrm{CDCl}_{3}, 400 \mathrm{MHz}\right) \delta 7.25(\mathrm{~m}, 5 \mathrm{H}),, 6.56(\mathrm{~d}, \mathrm{~J}=15.88$ $\mathrm{Hz}, 1 \mathrm{H}), 6.20(\mathrm{dt}, \mathrm{J}=6.4 \mathrm{~Hz}, 1 \mathrm{H}), 4.65(\mathrm{~d}, \mathrm{~J}=6.4 \mathrm{~Hz}, 2 \mathrm{H}), 2.25(\mathrm{t}, \mathrm{J}=7.4 \mathrm{~Hz}$, $2 \mathrm{H}), 1.61(\mathrm{q}, \mathrm{J}=7.4 \mathrm{~Hz}, 2 \mathrm{H}), 0.88(\mathrm{t}, \mathrm{J}=7.4 \mathrm{~Hz}, 3 \mathrm{H}) \cdot{ }^{13} \mathrm{C} \mathrm{NMR}\left(\mathrm{CDCl}_{3}, 100\right.$ $\mathrm{MHz}) 173.3,136.2,134.9,128.5,127.9,126.5,123.3,64.7,36.1,18.4$, 13.6;

Compound 3c, ${ }^{1} \mathrm{H}$ NMR $\left(\mathrm{CDCl}_{3}, 400 \mathrm{MHz}\right) \delta 7.25(\mathrm{~m}, 5 \mathrm{H}), 6.56(\mathrm{~d}, \mathrm{~J}=15.88$ $\mathrm{Hz}, 1 \mathrm{H}), 6.20(\mathrm{dt}, \mathrm{J}=6.4 \mathrm{~Hz}, 1 \mathrm{H}), 4.65(\mathrm{~d}, \mathrm{~J}=6.4 \mathrm{~Hz}, 2 \mathrm{H}), 2.27(\mathrm{t}, \mathrm{J}=7.48 \mathrm{~Hz}$, $2 \mathrm{H}), 1.58(\mathrm{t}, \mathrm{J}=7.4 \mathrm{~Hz}, 2 \mathrm{H}), 1.24(\mathrm{~m}, 4 \mathrm{H}), 0.81(\mathrm{~m}, 3 \mathrm{H}) .{ }^{13} \mathrm{C}$ NMR $\left(\mathrm{CDCl}_{3}, 100\right.$ $\mathrm{MHz}) \delta 173.5,136.2,133.9,128.5,127.9,126.5,126.0,123.3,64.7,34.2,31.2$, 24.6, 22.2, 13.8;

Compound 3d, ${ }^{1} \mathrm{H}$ NMR $\left(\mathrm{CDCl}_{3}, 400 \mathrm{MHz}\right) \delta 7.22(\mathrm{~m}, 5 \mathrm{H}), 6.57(\mathrm{~d}, \mathrm{~J}=15.92$ $\mathrm{Hz}, 1 \mathrm{H}), 6.20(\mathrm{dt}, \mathrm{J}=6.44 \mathrm{~Hz}, 1 \mathrm{H}), 5.48(\mathrm{q}, \mathrm{J}=5.36 \mathrm{~Hz}, 1 \mathrm{H}), 4.66(\mathrm{~d}, \mathrm{~J}=6.44 \mathrm{~Hz}$, $1 \mathrm{H}), 2.99(\mathrm{~d}, \mathrm{~J}=5.4 \mathrm{~Hz}, 2 \mathrm{H}), 1.96(\mathrm{~m}, 2 \mathrm{H}), 1.25(\mathrm{~m}, 6 \mathrm{H}), 0.80(\mathrm{t}, \mathrm{J}=6.40 \mathrm{~Hz}$, $3 \mathrm{H}) .{ }^{13} \mathrm{C} \mathrm{NMR}\left(\mathrm{CDCl}_{3}, 100 \mathrm{MHz}\right) \delta 173.9,135.0,134.1,128.6,126.6,123.2,121.3$, 65.1, 38.1, 32.1, 31.3, 22.1, 13.9;

Compound 3e, ${ }^{1} \mathrm{H}$ NMR $\left(\mathrm{CDCl}_{3}, 400 \mathrm{MHz}\right) \delta 7.81-7.24(\mathrm{~m}, 13 \mathrm{H}),, 6.35(\mathrm{~d}, \mathrm{~J}$ $=15.88 \mathrm{~Hz}, 1 \mathrm{H}), 5.89(\mathrm{dt}, \mathrm{J}=6.4 \mathrm{~Hz}, 1 \mathrm{H}), 4.63(\mathrm{~d}, \mathrm{~J}=6.4 \mathrm{~Hz}, 2 \mathrm{H}) .{ }^{13} \mathrm{C} \mathrm{NMR}$ $\left(\mathrm{CDCl}_{3}, 100 \mathrm{MHz}\right) \delta 168.4,142.4,141.3,136.2,133.8,131.2,130.6,129.7,128.4$, $128.3,128.0,127.8,127.1,126.5,122.5,65.3$;

Compound 3f, ${ }^{1} \mathrm{H}$ NMR $\left(\mathrm{CDCl}_{3}, 400 \mathrm{MHz}\right) \delta 7.49-7.31(\mathrm{~m}, 8 \mathrm{H}), 6.81(\mathrm{~d}, \mathrm{~J}=$ $15.88 \mathrm{~Hz}, 1 \mathrm{H}), 6.45(\mathrm{dt}, \mathrm{J}=6.44 \mathrm{~Hz}, 1 \mathrm{H}), 5.04(\mathrm{dt}, \mathrm{J}=6.44 \mathrm{~Hz}, 2 \mathrm{H}) .{ }^{13} \mathrm{C} \mathrm{NMR}$ $\left(\mathrm{CDCl}_{3}, 100 \mathrm{MHz}\right) \delta 165.4,136.2,134.7,132.6,131.5,128.6,128.2,126.7,122.8$, 66.1;

Compound 3g, ${ }^{1} \mathrm{H}$ NMR $\left(\mathrm{CDCl}_{3}, 400 \mathrm{MHz}\right) \delta 7.32-7.16(\mathrm{~m}, 7 \mathrm{H}), 6.65(\mathrm{~d}, \mathrm{~J}=$ $15.92 \mathrm{~Hz}, 1 \mathrm{H}), 6.29$ (dt, J = 7.48 Hz, 1H), 4.87 (d, J = 6.36 Hz, 2H), 2.23 (s, 3H). ${ }^{13} \mathrm{C} \mathrm{NMR}\left(\mathrm{CDCl}_{3}, 100 \mathrm{MHz}\right) \delta 166.5,143.7,134.1,129.7,128.8,128.6,127.9$, 126.6, 126.1, 123.4, 65.3, 21.7;

Compound 3h, ${ }^{1} \mathrm{H}$ NMR $\left(\mathrm{CDCl}_{3}, 400 \mathrm{MHz}\right) \delta 7.63-7.28(\mathrm{~m}, 10 \mathrm{H}), 6.81(\mathrm{~d}, \mathrm{~J}$ $=15.88 \mathrm{~Hz}, 1 \mathrm{H}), 6.46(\mathrm{dt}, \mathrm{J}=6.4 \mathrm{~Hz}, 1 \mathrm{H}), 5.04(\mathrm{~d}, \mathrm{~J}=6.4 \mathrm{~Hz}, 2 \mathrm{H}) .{ }^{13} \mathrm{C} \mathrm{NMR}$ $\left(\mathrm{CDCl}_{3}, 100 \mathrm{MHz}\right) \delta 166.4,134.3,133.0,129.7,128.6,128.4,128.1,127.9,126.1$, 123.3, 65.5;

Compound 3i, ${ }^{1} \mathrm{H}$ NMR $\left(\mathrm{CDCl}_{3}, 400 \mathrm{MHz}\right) \delta 7.49-7.01(\mathrm{~m}, 9 \mathrm{H}), 6.63(\mathrm{~d}, \mathrm{~J}=$ $15.88 \mathrm{~Hz}, 1 \mathrm{H}), 6.28(\mathrm{~m}, 1 \mathrm{H}), 4.84(\mathrm{~d}, \mathrm{~J}=6.48,2 \mathrm{H}) .{ }^{13} \mathrm{C} \mathrm{NMR}\left(\mathrm{CDCl}_{3}, 100 \mathrm{MHz}\right)$ $\delta 169.1,164.3,150.6,140.0,134.6,133.9,131.9,128.8,128.6,127.9,126.6,123.8$, 122.8, 65.7, 21.0;

Compound 3j, ${ }^{1} \mathrm{H} \mathrm{NMR}\left(\mathrm{CDCl}_{3}, 400 \mathrm{MHz}\right) \delta 7.65(\mathrm{~d}, \mathrm{~J}=16.0 \mathrm{~Hz}, 1 \mathrm{H}), 7.34-$ $7.15(\mathrm{~m}, 10 \mathrm{H}), 6.62(\mathrm{~d}, \mathrm{~J}=15.88 \mathrm{~Hz}, 1 \mathrm{H}), 6.40(\mathrm{~d}, \mathrm{~J}=16.0 \mathrm{~Hz}, 1 \mathrm{H}), 6.28(\mathrm{dt}, \mathrm{J}=$ $6.48 \mathrm{~Hz}, 1 \mathrm{H}), 4.78(\mathrm{~d}, \mathrm{~J}=6.44 \mathrm{~Hz}, 2 \mathrm{H}) ;{ }^{13} \mathrm{C}$ NMR (Acetone-D6, $\left.100 \mathrm{MHz}\right) \delta$ 171.0, 149.8, 141.6, 139.6, 138.2, 135.4, 134.0, 133.3, 131.6, 128.9, 123.1, 69.7.

\subsection{Figure 2}

Compound 3k, ${ }^{1} \mathrm{H}$ NMR $\left(\mathrm{CDCl}_{3}, 400 \mathrm{MHz}\right) \delta 5.46(\mathrm{~m}, 1 \mathrm{H}), 5.27(\mathrm{~m}, 1 \mathrm{H}), 4.16$ $(\mathrm{d}, \mathrm{J}=6.4 \mathrm{~Hz}, 2 \mathrm{H}), 1.99(\mathrm{q}, \mathrm{J}=7.52 \mathrm{~Hz}, 2 \mathrm{H}), 1.40(\mathrm{~d}, \mathrm{~J}=6.52,3 \mathrm{H}) ;{ }^{13} \mathrm{C} \mathrm{NMR}$ $\left(\mathrm{CDCl}_{3}, 100 \mathrm{MHz}\right) \delta 173.2,130.1,125.2,64.3,29.9,26.8,17.0,8.49$; 
Compound 31, ${ }^{1} \mathrm{H} \mathrm{NMR}\left(\mathrm{CDCl}_{3}, 400 \mathrm{MHz}\right) \delta 5.53(\mathrm{~m}, 1 \mathrm{H}), 5.49(\mathrm{~m}, 1 \mathrm{H}), 4.42$ $(\mathrm{d}, \mathrm{J}=6.42 \mathrm{~Hz}, 2 \mathrm{H}), 2.23(\mathrm{t}, \mathrm{J}=7.64 \mathrm{~Hz}, 2 \mathrm{H}), 1.65(\mathrm{~d}, \mathrm{~J}=6.44 \mathrm{~Hz}, 3 \mathrm{H}), 1.55(\mathrm{~m}$, $2 \mathrm{H}), 1.23(\mathrm{~m}, 4 \mathrm{H}), 0.82(\mathrm{t}, \mathrm{J}=6.8 \mathrm{~Hz}, 3 \mathrm{H}) .{ }^{13} \mathrm{C} \mathrm{NMR}\left(\mathrm{CDCl}_{3}, 100 \mathrm{MHz}\right) \delta 173.5$, 131.0, 125.1, 64.9, 34.2, 31.2, 22.2, 17.0, 13.7;

Compound $3 \mathrm{~m},{ }^{1} \mathrm{H}$ NMR $\left(\mathrm{CDCl}_{3}, 400 \mathrm{MHz}\right) \delta 5.78(\mathrm{~m}, 1 \mathrm{H}), 5.51(\mathrm{~m}, 2 \mathrm{H})$, $4.48(\mathrm{~d}, \mathrm{~J}=6.48 \mathrm{~Hz}, 1 \mathrm{H}), 3.00(\mathrm{~d}, \mathrm{~J}=5.44 \mathrm{~Hz}, 2 \mathrm{H}), 2.00(\mathrm{~m}, 2 \mathrm{H}), 1.69(\mathrm{~m}, 3 \mathrm{H})$, $1.29(\mathrm{~m}, 6 \mathrm{H}), 0.86(\mathrm{t}, \mathrm{J}=7.0,3 \mathrm{H}) .{ }^{13} \mathrm{C} \mathrm{NMR}\left(\mathrm{CDCl}_{3}, 100 \mathrm{MHz}\right) \delta 172.0,134.8$, 131.3, 125.121.4, 65.2, 38.1, 32.1, 331.3, 22.1, 17.7, 13.8;

Compound 3n, ${ }^{1} \mathrm{H}$ NMR $\left(\mathrm{CDCl}_{3}, 400 \mathrm{MHz}\right) \delta 7.83-7.32(\mathrm{~m}, 9 \mathrm{H}), 5.5(\mathrm{~m}$, $1 \mathrm{H}), 5.27(\mathrm{~m}, 1 \mathrm{H}), 4.47(\mathrm{~d}, \mathrm{~J}=6.52,2 \mathrm{H}), 1.08(\mathrm{~d}, \mathrm{~J}=6.48,3 \mathrm{H}) .{ }^{13} \mathrm{C} \mathrm{NMR}\left(\mathrm{CDCl}_{3}\right.$, $100 \mathrm{MHz}) \delta 166.5,142.4,141.4,137.3,131.2$, 130.6, 129.7, 128.5, 128.0, 127.1, $124.5,60.5,17.7$;

Compound 3o, ${ }^{1} \mathrm{H}$ NMR $\left(\mathrm{CDCl}_{3}, 400 \mathrm{MHz}\right) \delta 7.75-7.18(\mathrm{~m}, 4 \mathrm{H}), 5.80(\mathrm{~m}$, $1 \mathrm{H}), 5.63(\mathrm{~m}, 1 \mathrm{H}), 4.69(\mathrm{~d}, \mathrm{~J}=6.4 \mathrm{~Hz}, 2 \mathrm{H}), 1.68(\mathrm{~d}, \mathrm{~J}=6.0 \mathrm{~Hz}, 3 \mathrm{H}) .{ }^{13} \mathrm{C} \mathrm{NMR}$ $\left(\mathrm{CDCl}_{3}, 100 \mathrm{MHz}\right) \mathrm{d} 165.5,137.3,133.6,132.4,131.9,131.0,126.5,124.7,116.3$, $66.2,17.8$;

Compound 3p, ${ }^{1} \mathrm{H}$ NMR $\left(\mathrm{CDCl}_{3}, 400 \mathrm{MHz}\right) \delta 7.91(\mathrm{~d}, \mathrm{~J}=8.2 \mathrm{~Hz}, 2 \mathrm{H}), 7.21(\mathrm{~d}$, $\mathrm{J}=8.0 \mathrm{~Hz}, 2 \mathrm{H}), 5.58(\mathrm{~m}, 1 \mathrm{H}), 5.68(\mathrm{~m}, 1 \mathrm{H}), 4.71(\mathrm{~d}, \mathrm{~J}=6.36 \mathrm{~Hz}, 2 \mathrm{H}), 2.38$ (s, $3 \mathrm{H}), 1.72(\mathrm{~d}, \mathrm{~J}=6.2 \mathrm{~Hz}, 3 \mathrm{H}), 0.80(\mathrm{t}, \mathrm{J}=6.0 \mathrm{~Hz}, 3 \mathrm{H}) \cdot{ }^{13} \mathrm{C} \mathrm{NMR}\left(\mathrm{CDCl}_{3}, 100\right.$ $\mathrm{MHz}) \delta 166.5,143.5,131.1,129.6,127.6,125.2,124.4,65.4,17.8$;

Compound 3q, ${ }^{1} \mathrm{H}$ NMR $\left(\mathrm{CDCl}_{3}, 400 \mathrm{MHz}\right) \delta 8.02-7.49(\mathrm{~m}, 5 \mathrm{H}), 5.89(\mathrm{~m}$, $1 \mathrm{H}), 5.73(\mathrm{~m}, 1 \mathrm{H}), 4.74(\mathrm{~d}, \mathrm{~J}=6.32,2 \mathrm{H}), 1.74(\mathrm{~d}, \mathrm{~J}=6.3 \mathrm{~Hz}, 3 \mathrm{H}) .{ }^{13} \mathrm{C} \mathrm{NMR}$ $\left(\mathrm{CDCl}_{3}, 100 \mathrm{MHz}\right) \delta 162.1,133.9,131.7,130.2,126.4,66.0,17.9$;

Compound 3r, ${ }^{1} \mathrm{H}$ NMR $\left(\mathrm{CDCl}_{3}, 400 \mathrm{MHz}\right) \delta 8.00-7.06(\mathrm{~m}, 4 \mathrm{H}), 5.85(\mathrm{~m}$, $1 \mathrm{H}), 5.65(\mathrm{~m}, 1 \mathrm{H}), 4.67(\mathrm{~d}, \mathrm{~J}=6.52 \mathrm{~Hz}, 2 \mathrm{H}), 2.30(\mathrm{~s}, 3 \mathrm{H}), 1.72(\mathrm{~d}, \mathrm{~J}=6.52 \mathrm{~Hz}$, $3 \mathrm{H}) .{ }^{13} \mathrm{C} \mathrm{NMR}\left(\mathrm{CDCl}_{3}, 100 \mathrm{MHz}\right) \delta 169.6,164.3,150.6,133.7,131.8,125.9,124.8$, 123.7, 65.8, 21.0, 17.7;

Compound 3s, ${ }^{1} \mathrm{H} \mathrm{NMR}\left(\mathrm{CDCl}_{3}, 400 \mathrm{MHz}\right) \delta 7.70-7.42(\mathrm{~m}, 5 \mathrm{H}, 1 \mathrm{H}), 6.55(\mathrm{~d}$, $\mathrm{J}=16.06 \mathrm{~Hz}, 1 \mathrm{H}), 5.76(\mathrm{~m}, 2 \mathrm{H}), 4.60(\mathrm{~d}, \mathrm{~J}=6.4 \mathrm{~Hz}, 2 \mathrm{H}), 1.7(\mathrm{~m}, 3 \mathrm{H})$;

Compound 4a, ${ }^{1} \mathrm{H}$ NMR $\left(\mathrm{CDCl}_{3}, 400 \mathrm{MHz}\right) \delta 7.23(\mathrm{~m}, 5 \mathrm{H}), 6.56(\mathrm{~d}, \mathrm{~J}=13.4$ $\mathrm{Hz}, 1 \mathrm{H}), 6.20(\mathrm{dt}, \mathrm{J}=6.44 \mathrm{~Hz}, 1 \mathrm{H}), 5.28(\mathrm{~m}, 8 \mathrm{H}), 4.64(\mathrm{~d}, \mathrm{~J}=6.48 \mathrm{~Hz}, 2 \mathrm{H}), 2.72$ $(\mathrm{m}, 6 \mathrm{H}), 2.29(\mathrm{t}, \mathrm{J}=7.6 \mathrm{~Hz}, 2 \mathrm{H}), 1.98(\mathrm{~m}, 4 \mathrm{H}), 1.65(\mathrm{t}, \mathrm{J}=7.4 \mathrm{~Hz}, 2 \mathrm{H}), 1.20(\mathrm{~m}$, $6 \mathrm{H}), 0.80$ (m, 3H);

Compound 4b, ${ }^{1} \mathrm{H}$ NMR $\left(\mathrm{CDCl}_{3}, 400 \mathrm{MHz}\right) \delta 5.72(\mathrm{~m}, 1 \mathrm{H}), 5.56(\mathrm{~m}, 1 \mathrm{H}), 5.35$ $(\mathrm{m}, 8 \mathrm{H}), 4.47(\mathrm{~d}, 6.5 \mathrm{~Hz}, 2 \mathrm{H}), 2.77(\mathrm{~m}, 6 \mathrm{H}), 2.30(\mathrm{~m}, 2 \mathrm{H}), 2.08(\mathrm{~m}, 4 \mathrm{H}), 1.75(\mathrm{~m}$, $2 \mathrm{H}, 3 \mathrm{H}), 1.27(\mathrm{~m}, 6 \mathrm{H}), 0.86(\mathrm{~m}, 3 \mathrm{H})$;

Compound 4c, major peak: ${ }^{1} \mathrm{H} \mathrm{NMR}\left(\mathrm{CDCl}_{3}, 400 \mathrm{MHz}\right) \delta 9.87(\mathrm{~s}, 1 \mathrm{H}), 9.56$ (s, $1 \mathrm{H}), 9.54(\mathrm{~s}, 1 \mathrm{H}), 7.17$ (m, aromatic), 6.5 - 6.25 (d, dt), 4.97 (m, 2H X 2).

\section{Acknowledgements}

ArponaHira gratefully acknowledges the graduate fellowship award from Tennessee State University, Nashville, TN. Authors thankfully acknowledge the NMR assistance from Dr. Donald F. Stec, Vanderbilt University, Nashville, TN. 


\section{Conflicts of Interest}

The authors declare no conflicts of interest regarding the publication of this paper.

\section{References}

[1] Marshall, J.A. (2000) Synthesis and Reactions of Allylic, Allenic, Vinylic, and Arylmetal Reagents from Halides and Esters via Transient Organopalladium Intermediates. Chemical Reviews, 100, 3163-3185. https://doi.org/10.1021/cr000003u

[2] Negishi, E., Huang, Z., Wang, G., Mohan, S., Wang, C. and Hattori, H. (2008) Recent Advances in Efficient and Selective Synthesis of Di-, Tri-, and Tetrasubstituted Alkenes via Pd-Catalyzed Alkenylation-Carbonyl Olefination Synergy. Accounts of Chemical Research, 41, 1474-1485. https://doi.org/10.1021/ar800038e

[3] Okamoto, N., Miwa, Y., Minami, H., Takeda, K. and Yanada, R. (2011) Regio- and Stereoselective Multisubstituted Enol Ester Synthesis. The Journal of Organic Chemistry, 76, 9133-9138. https://doi.org/10.1021/ar800038e

[4] Satchell, D.P.N. (1963) An Outline of Acylation. Quarterly Reviews, Chemical Society, 17, 160. https://doi.org/10.1039/qr9631700160

[5] Eftekhari-Sis, B. and Zirak, M. (2017) $\alpha$-Imino Esters in Organic Synthesis: Recent Advances. Chemical Reviews, 117, 8326-8419. https://doi.org/10.1021/acs.chemrev.7b00064

[6] Inanaga, J., Kirata, K., Saeki, H., Katsuki, T. and Yamaguchi, M. (1979) A Rapis Esterification by Means of Mixed Anhydride and Its Application to Large-Ring Lactonization. Bulletin of the Chemical Society of Japan, 52, 1989-1993.

https://doi.org/10.1246/bcsj.52.1989

[7] Fischer, E. (1895) Darstellung der Ester. Berichte der Deutschen Chemischen Gesellschaft, 28, 3252-3258. https://doi.org/10.1002/cber.189502803176

[8] Grasa, G.A. and Colacot, T.J. (2007) $\alpha$-Arylation of Ketones Using Highly Active, Air-Stable ( $\left.\mathrm{D}^{\mathrm{t}} \mathrm{BPF}\right) \mathrm{PdX}_{2}(\mathrm{X}=\mathrm{Cl}, \mathrm{Br})$ Catalysts. Organic Letters, 9, 5489-5492.

[9] Al-Masum, M., Saleh, N. and Islam, T. (2013) A Novel Route to Organonitrites by Pd-Catalyzed Cross-Coupling of Sodium Nitriteand Potassium Organotrifluoroborates. Tetrahedron Letters, 54, 1141-1144.

[10] Mann, G., Shelby, Q., Roy, A.H. and Hartwig, J.F. (2003) Electronic and Steric Effects on the Reductive Elimination of Diaryl Ethers from Palladium(II). Organometallics, 22, 2775-2789. https://doi.org/10.1021/om030230x

[11] Elsagir, A.R., Gassner, F., Gorls, H. and Dinjus, E. (2000) Bidentate Ferrocenyl Phosphines and Their Palladium(II)Dichloride Complexes-X-Ray Structural and NMR Spectroscopic Investigations and First Results of Their Characteristics in the Pd-Catalyzed Oligomerization of 1,3-Butadiene with $\mathrm{CO}_{2}$. Journal of Organometallic Chemistry, 597, 139-145. https://doi.org/10.1016/S0022-328X(99)00670-1

[12] Bianchini, C., Meli, A., Overhauser, W., Parisel, S., Passaglia, E., Ciardelli, F., Gusev, O.V., Kal'sin, A.M. and Vologdin, N.V. (2005) Ethylene Carbonylation in Methanol and in Aqueous Media by Palladium(II) Catalysts Modified with 1,1'Bis(Dialkylphosphino)Ferrocenes. Organometallics, 24, 1018-1030. https://doi.org/10.1021/om049109w

[13] Martin, S.A., Brash, A.R. and Murphy, R.C. (2016) The Discovery and Early Structural Studies of Arachidonic Acid. The Journal of Lipid Research, 57, 1126-1132. https://doi.org/10.1194/jlr.R068072 
[14] Li, D., Ng, A., Mann, N.J. and Sinclair, A.J. (1998) Contribution of Meat Fat to Dietary Arachidonic Acid. Lipids, 33, 437-440. https://doi.org/10.1007/s11745-998-0225-7

[15] Tallima, H. and Rashika, E.R. (2018) Arachidonic Acid: Physiological Roles and Potential Health Benefits-A Review. Journal of Advanced Research, 4, 467-468. https://doi.org/10.1016/j.jare.2017.11.004

[16] Groehn, V., Moser, R. and Pugin, B. (2005) Stereoselective Hydrogenation of Folic Acid Dimethyl Ester Benzenesulfonate: A New Access to Optically Pure 1-Tetrahydrofolic Acid. Advanced Synthesis \& Catalysis, 347, 1855-1862. https://doi.org/10.1002/adsc.200505098

[17] Bailey, L.B. (1995) Folate in Health and Disease. Vol. 1, Marcel Dekker, Inc., New York, Basel, Hong Kong, 23-42. 